\title{
The effects of traditional, superset, and tri-set resistance training structures on perceived intensity and physiological responses
}

\author{
Jonathon J. S. Weakley ${ }^{1,2}$ - Kevin Till ${ }^{1,2} \cdot$ Dale B. Read ${ }^{1,2} \cdot$ Gregory A. B. Roe $^{1,2}$ • \\ Joshua Darrall-Jones $^{1,2}$ - Padraic J. Phibbs ${ }^{1,2} \cdot$ Ben Jones $^{1,2,3}$
}

Received: 17 May 2017 / Accepted: 5 July 2017 / Published online: 11 July 2017

(C) The Author(s) 2017. This article is an open access publication

\begin{abstract}
Purpose Investigate the acute and short-term (i.e., $24 \mathrm{~h}$ ) effects of traditional (TRAD), superset (SS), and tri-set (TRI) resistance training protocols on perceptions of intensity and physiological responses.

Methods Fourteen male participants completed a familiarisation session and three resistance training protocols (i.e., TRAD, SS, and TRI) in a randomised-crossover design. Rating of perceived exertion, lactate concentration ([Lac]), creatine kinase concentration $([\mathrm{CK}])$, countermovement jump (CMJ), testosterone, and cortisol concentrations was measured pre, immediately, and 24-h post the resistance training sessions with magnitude-based inferences assessing changes/differences within/between protocols.

Results TRI reported possible to almost certainly greater efficiency and rate of perceived exertion, although session perceived load was very likely lower. SS and TRI had very likely to almost certainly greater lactate responses during the protocols, with changes in [CK] being very likely and likely increased at $24 \mathrm{~h}$, respectively. At 24-h post-training, CMJ variables in the TRAD protocol had returned to baseline; however, SS and TRI were still possibly to likely reduced. Possible increases in testosterone immediately
\end{abstract}

Communicated by William J. Kraemer.

Jonathon J. S. Weakley

j.j.weakley@leedsbeckett.ac.uk

1 Room G03, Macaulay Hall, Institute for Sport, Physical Activity and Leisure, Centre for Sports Performance, Headingley Campus, Leeds Beckett University, West Yorkshire LS6 3GZ, UK

2 Yorkshire Carnegie Rugby Union Club, Kirkstall Training Ground, Leeds Rugby Academy, Leeds, West Yorkshire, UK

3 The Rugby Football League, Leeds, West Yorkshire, UK post SS and TRI protocols were reported, with SS showing possible increases at 24-h post-training. TRAD and SS showed almost certain and likely decreases in cortisol immediately post, respectively, with TRAD reporting likely decreases at 24-h post-training.

Conclusions SS and TRI can enhance training efficiency and reduce training time. However, acute and short-term physiological responses differ between protocols. Athletes can utilise SS and TRI resistance training, but may require additional recovery post-training to minimise effects of fatigue.

Keywords Efficiency $\cdot$ Resistance training · Countermovement jump · Testosterone $\cdot$ Cortisol $\cdot$ Lactate

\begin{tabular}{|c|c|}
\hline \multicolumn{2}{|c|}{ Abbreviations } \\
\hline TRAD & Traditional \\
\hline SS & Supersets \\
\hline TRI & Tri-sets \\
\hline [LAC] & Lactate \\
\hline$[\mathrm{CK}]$ & Creatine kinase concentration \\
\hline CMJ & Countermovement jump \\
\hline RPE & Rating of perceived exertion \\
\hline $3 \mathrm{RM}$ & Three repetition maximum \\
\hline FT:CT & Flight time:contraction time \\
\hline PP/BM & Peak power per kilogram of body mass \\
\hline $\mathrm{kg} \min ^{-1}$ & Volume load completed per minute \\
\hline SWC/D & Smallest worthwhile change/difference \\
\hline ES & Effect size \\
\hline
\end{tabular}

\section{Introduction}

Resistance training is known to improve measures of strength, power, and lean body mass (Pareja-Blanco et al. 
2016). Furthermore, it is established that it can enhance physical performance (e.g., jump height) which may benefit sporting outcomes (Pareja-Blanco et al. 2016). However, athletes are often unable to commit prolonged periods of time to resistance training due to other training requirements (e.g., skill development and other conditioning priorities) (Phibbs et al. 2017). Therefore, resistance training protocols such as supersets (SS) (i.e., the completion of two exercises consecutively followed by a recovery period) and tri-sets (TRI) (i.e., the completion of three exercises consecutively followed by a recovery period) that enhance training efficiency (i.e., kilograms lifted per minute) may be an effective mechanism to provide an appropriate resistance training stimulus, in a short period of time (Sabido et al. 2016; Schoenfeld 2011).

The use of SS and TRI has been proposed as an efficacious method of enhancing strength and body composition (Robbins et al. 2010a, b). In studies that have investigated these training protocols, there does not appear to be any detrimental effect on resistance training volume despite the reduction in recovery time within each training session (Maia et al. 2014; Robbins et al. 2010a). However, this reduction in training time may alter perceptions of internal training load [i.e., rating of perceived exertion (RPE)] (Balsamo et al. 2012). To this end, SS and TRI can improve training efficiency, reduce training time, and alter perceived training load, although the magnitude of these changes is still unknown.

In addition to reduced training time and altered perception of intensity, resistance training methods that have varying rates of efficiency are also known to promote divergent metabolic and endocrine responses (Hiscock et al. 2017; Schoenfeld 2010; Walker et al. 2011). It has been suggested that the cause of this variation is in part a result of the resistance training efficiency imposed (McCaulley et al. 2009). It has been postulated that protocols that complete large amounts of volume within a given time elicit greater metabolic responses (Hooper et al. 2017). This increase in metabolic perturbation, evidenced by lowered $\mathrm{pH}$ and higher lactate concentrations ([LAC]), may then provoke augmented endocrine outcomes (i.e., increased testosterone and cortisol secretion) (Hooper et al. 2017). While previous research has examined relationships between resistance training efficiency, and metabolic and endocrine responses (Hiscock et al. 2017; McCaulley et al. 2009), the effect of enhanced efficiency due to SS and TRI resistance training structuring has not been considered. To this end, the physiological responses due to these training methods are not well understood. Furthermore, changes in training efficiency are also known to affect neuromuscular function (Hiscock et al. 2017). McCaulley et al. (2009) previously suggested that increased resistance training efficiency may cause a reliance upon anaerobic glycolysis and an accumulation of metabolites (i.e., [LAC]) which may damage contractile properties within the muscle (McCaulley et al. 2009). These changes may potentially cause reduced force-generating capacity and impact upon subsequent neuromuscular performance [e.g., countermovement jump (CMJ)]. However, volume and intensity controlled trials have not assessed the magnitude and short-term (i.e., $24 \mathrm{~h}$ ) effects of increased training efficiency upon neuromuscular responses.

Establishing resistance training protocols and structures that effectively utilise an athlete's time is of benefit. SS and TRI have previously been established as two forms of resistance training that manage this (Robbins et al. 2010a). However, the acute and short-term physiological responses to these methods of resistance training have not yet been established. Consequently, investigation of these training methods will provide an improved understanding of how enhanced training efficiency can affect perception of training load, metabolic and muscle damage outcomes, endocrine responses, and neuromuscular function following different resistance set structures. Therefore, the aim of this study was to investigate the acute and short-term (i.e., $24 \mathrm{~h}$ ) effects of traditional (TRAD), SS, and TRI volume and intensity equated resistance training protocols on perception of training intensity, and metabolic, neuromuscular, and endocrine responses in well-trained male athletes.

\section{Methods}

\section{Participants}

Fourteen male university rugby union players with a resistance training history of over 2 years were recruited to take part in the study (Table 1). All participants had at least 6 months uninterrupted resistance training, performing at least three resistance training sessions each week. Participants were screened prior to the study for any contraindication to physical activity. Throughout the study participants refrained from dietary supplements, and the only medication used by one participant was for the treatment of mild asthma. All protocols were explained and informed consent was obtained from all individual participants included in the study prior to the beginning of the study. Ethics approval was granted by the university ethics committee.

\section{Experimental design}

This study was a randomised-crossover design that took place over 4 weeks at the beginning of the university rugby 
Table 1 Mean \pm SD of participant anthropometric and physical characteristics

\begin{tabular}{lclr}
\hline Age (years) & $20.8 \pm 1.2$ & Bench press 3RM $(\mathrm{kg})$ & $105.2 \pm 15.2$ \\
Height $(\mathrm{m})$ & $1.81 \pm 0.06$ & Romanian deadlift 3RM $(\mathrm{kg})$ & $143.2 \pm 30.8$ \\
Body mass $(\mathrm{kg})$ & $87.3 \pm 6.2$ & Dumbbell shoulder press 3RM $(\mathrm{kg})$ & $66.0 \pm 8.6$ \\
Training age (years) & $4.1 \pm 1.2$ & Bent-over row 3RM $(\mathrm{kg})$ & $95.0 \pm 14.5$ \\
Back squat 3RM $(\mathrm{kg})$ & $141.1 \pm 31.9$ & Upright row 3RM $(\mathrm{kg})$ & $60.1 \pm 6.9$ \\
\hline
\end{tabular}

union pre-season (i.e., July-August). All participants had followed an off-season resistance training programme and initiated the study with a comparable training base. The study consisted of a familiarisation assessment, followed by three resistance training protocols (i.e., TRAD, SS, TRI) in a randomised order. Twenty-four hours after the resistance training protocol participants were required to return to the exercise laboratory for follow-up measures. Figure 1 further outlines the protocols and timepoints. In the $48 \mathrm{~h}$ before all testing, participants were asked to refrain from vigorous exercise, maintain normal dietary patterns, sleep well (i.e., $>7 \mathrm{~h}$ ), consume a meal $2 \mathrm{~h}$ before testing, and maintain normal hydration levels. After each testing session, participants were asked to consume their regular postexercise meal and to not partake in any further exercise for $24 \mathrm{~h}$.

\section{Resistance training protocols}

Each protocol contained the same six exercises (i.e., back squat, bench press, Romanian deadlift, dumbbell shoulder press, bent-over row, and upright row), although grouped into a TRAD, SS, or TRI configuration (refer to Fig. 1). TRAD resistance training referred to the completion of a single exercise set followed by a rest period. All exercise sets were then completed prior to completing any other exercises. In the SS protocol, however, two different exercise sets were completed consecutively followed by a rest period. These two different exercises were then repeated until the required number of sets was completed. TRI involved an additional exercise and set (i.e., three consecutive sets of different exercises) followed by a rest period. All three exercise sets were then completed prior to completing the final three exercises. Sixty-five percent of each movement's three repetition maximum (3RM) was calculated and used as the prescribed intensity for each exercise across the three training protocols. This intensity was selected due to previous research by Sabido et al. (2016) indicating that when completing supersets, intensities above this cause notable losses in repetition completion (i.e., 12.5\%). All participants participated in the three resistance training sessions with exactly 7 days between protocols.

On testing days, participants were informed of the protocol that would be completed, and provided a salivary and finger-tip blood sample on arrival for the analysis of testosterone and cortisol concentration, and [Lac] and creatine kinase concentration $[\mathrm{CK}]$, respectively. Upon the completion of a standardised warm up, which included dynamic exercise and exercise specific stretches [i.e., walking lunges, squats, heel flicks, high knees, skipping, three submaximal CMJ, and plyometric push ups (Roe et al. 2016b)], a CMJ was completed upon a force platform (NMP Technologies Ltd., ForceDecks Model FD4000a, London, UK). After the CMJ, an exercise specific warm up utilising the first exercise/grouping of exercises (i.e., squat in the TRAD; squat and bench press in the SS; squat, bench press, and Romanian deadlift in the TRI) was completed. This consisted of eight repetitions with an empty bar, followed by two sets

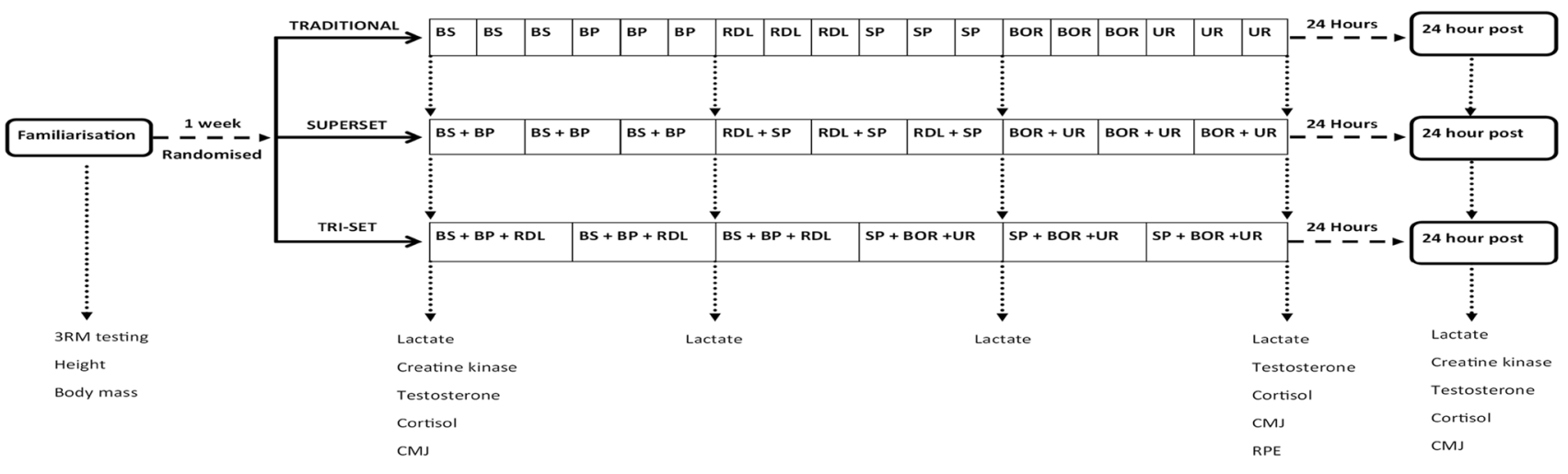

Fig. 1 Diagram outlining the order of experimental procedures. $3 R M$ three repetition maximum, $C M J$ countermovement jump, $R P E$ rate of perceived exertion, $B S$ back squat, $B P$ bench press, $R D L$ Romanian deadlift, $S P$ shoulder press, $B O R$ bent-over row, $U R$ upright row 
of five repetitions and then three repetitions at submaximal self-selected loads. This has previously been completed in resistance training literature (Darrall-Jones et al. 2015; Weakley et al. 2017a, b). After the completion of the exercise specific warm up, the exercises were organised with $65 \%$ of the participants $3 \mathrm{RM}$ and a 2 -min rest prior to the commencement of exercise was provided.

Exercises were grouped, so that similar muscle groups were not completed consecutively (Sabido et al. 2016), and so that large muscle groups were exercised prior to smaller muscle groups (e.g., back squat followed by bench press) (Haff and Triplett 2015). The specific exercise routine was undertaken with 2-min rest between every exercise set/grouping of exercises. While all repetitions maintained a two second eccentric phase (monitored by the lead researcher) followed by an explosive concentric phase. In total, six exercises each consisting of three sets and ten repetitions were completed. Finger-tip blood samples were taken after the 6th and 12th sets for the assessment of [Lac]. At the completion of the 18th and final set, finger-tip samples were taken again (for [Lac]), along with $\mathrm{CMJ}$ and salivary sample (for testosterone and cortisol concentration). 15 min after the completion of each resistance training protocol participants provided an RPE utilising a modified-Borg scale (Singh et al. 2007). At 24-h post-protocol, participants returned and provided a $5 \mathrm{ml}$ salivary sample and a finger-tip blood sample was taken for [CK]. Following these measures, participants completed the same standardised warm up as the previous day and completed CMJ testing upon the same force plate. Figure 1 outlines the protocols and timepoints of testing.

\section{Protocols}

\section{Strength measures and anthropometric assessment}

During a familiarisation session 3RM strength, body mass, and height were measured. Body mass and height were measured to the nearest $0.1 \mathrm{~kg}$ and $0.1 \mathrm{~cm}$, respectively, using calibrated Seca Alpha (model 220, Germany) scales and Seca Alpha (model 213, Germany) stadiometer. Strength was measured with the back squat, bench press, Romanian deadlift, dumbbell shoulder press, bent-over row, and upright row being tested. These movements were chosen as they have previously been used in similar studies (Harries et al. 2016; Smart and Gill 2013; Till et al. 2015), while all participants were familiar with the movements as they had been in previous resistance training programmes.

3RM strength was assessed using the following exercise protocols, previously outlined (Haff and Triplett
2015; Smart and Gill 2013; Uribe et al. 2010; Weakley et al. 2017b). The back squat was completed with the bar resting on the upper trapezium with participants required to lower themselves, so that the top of the thigh was parallel with the floor. The bench press was completed with hand position at a self-selected width with the bar lowered to the chest and returned to a locked-out position. Romanian deadlift maintained a slight bend in the knee and the lowering of the bar until immediately below the patellar. Dumbbell shoulder press begun with the arms holding the dumbbells, so that the elbow was at a $90^{\circ}$ angle with the dumbbells in line with the cranium. The arms were extended and lowered, so that the arms returned to the $90^{\circ}$ elbow angle. The bent-over row was completed with an overhand grip which raised the bar to the lower sternum, while the torso was maintained parallel to the ground. The upright row required the participant to hold the barbell with a shoulder width grip; the bar was then raised to the nipple line and returned to the hang position without any additional leg drive.

\section{Hormone assessment}

Saliva samples were taken at three timepoints (i.e., at arrival, immediately post the completion of each protocol, and at the 24-h post-protocol follow-up) across the 24-h period. To standardise collection of all salivary samples, participants were asked not to eat or brush their teeth within 60 min of providing a sample. Furthermore, 15 min before initiating a passive drool, participants were provided $200 \mathrm{ml}$ of water. This was used to ensure minimal sample dilution and to remove food residue within the mouth. The first and last saliva samples were taken at the same time each day to assist in the control for circadian rhythm. Saliva was used due to its previous use in rugby union players, ease of compliance, low invasiveness, and ability to measure biologically active hormones (e.g., testosterone and cortisol) (Crewther and Cook 2010; Gaviglio et al. 2014).

A $5 \mathrm{ml}$ volume of saliva was deposited from the mouth into a $10 \mathrm{ml}$ cryovial and centrifuged within $60 \mathrm{~min}$ of provision. Centrifugation was at 3000 RPM for $15 \mathrm{~min}$ with the supernatant then being transferred by pipette to commercially available storage equipment (Salimetrics Cryovials, Salimetrics, CA, USA). This was then stored immediately at $-80{ }^{\circ} \mathrm{C}$ prior to analysis at a private commercial laboratory (Psychology Laboratory, Anglia Ruskin University, UK). The interassay coefficient of variation $(\mathrm{CV})$ for the hormone responses was reported to be $4.3 \%$ for testosterone and $7.2 \%$ for cortisol. 


\section{CMJ assessment}

Analysis of CMJ was completed using a force platform (NMP Technologies Ltd., ForceDecks Model FD4000a, London, UK) which sampled at a rate of $1000 \mathrm{~Hz}$. All participants performed three CMJs with feet placed approximately shoulder width apart and with hands placed on hips. Participants lowered themselves to a selfselected depth and jumped as high as possible. Between each maximal exertion, $60 \mathrm{~s}$ rest was provided (Weakley et al. 2017b). The outcome variables which were included in the analysis were: CMJ height (jump height), flight time:contraction time (FT:CT), and peak power per $\mathrm{kg}$ of body mass (PP/BM). Jump height was used due to its common use as a measure of lower body power (Roe et al. 2016b; Till et al. 2016; Weakley et al. 2017b). FT:CT was assessed as it provides the practitioner insight into movement strategies and has been suggested to be a valuable measure of fatigue due to it being derived from time-related variables (Gathercole et al. 2015; McGuigan 2017). PP/BM was analysed due to its very large relationship ( $r=0.81$ ) with ballistic capabilities (Hori et al. 2008). The best of the three scores at each timepoint was used in analysis (Haddad et al. 2015). Participant CV of these variables was reported to be $4.6 \%$ (jump height), 7.8\% (FT:CT), and PP/BM (1.3\%).

\section{Metabolic assessment}

Whole blood samples were collected to assess [CK] upon arrival at the testing facility and at the same corresponding timepoint $24 \mathrm{~h}$ later. Samples were collected via finger-tip puncture which was made with a spring-loaded single use disposable lancet. Approximately $30 \mu \mathrm{l}$ of whole capillary blood was collected using a plastic capillary tube (MICROSAFE(C, Safe-tec, Numbrecht, Ivyland, USA) and immediately analysed using reflectance photometry (Reflotron ${ }^{\circledR}$ Plus, Boehringer, Manheim, Germany). 20 min before each testing session, the machine was calibrated using a standardised $[\mathrm{CK}]$ strip. Participant reliability of this machine has previously been reported $(\mathrm{CV}=5.3 \%)$ (Roe et al. 2016a).

Blood [Lac] was analysed before, during, and after the exercise protocols using a lactate analyser (Lactate Plus, Nova Biomedical, MA, USA). After sterilising the finger, a puncture was made with a spring-loaded single use disposable lancet. The first drop of blood was wiped away, with the second drop being applied to an assay strip and inserted into the [LAC] analysing device. This device has previously been reported to demonstrate high levels of reliability (intraclass correlation coefficient $=0.99$ ) at a range of [Lac] values (Baldari et al. 2009).
RPE and session perceived load measures

Participants were asked to provide an RPE 15 min after each resistance training protocol after being asked the question "How was your workout?" Participants were presented with a modified-Borg ratio-10 scale and verbally indicated an answer which was recorded (Singh et al. 2007). Training time was recorded to the nearest minute of duration by the lead researcher, with this time being recorded and then multiplied with the corresponding RPE value to provide session perceived load (Foster et al. 2001).

\section{Calculation of volume load and efficiency}

Volume load (kg) [i.e., the multiplication of all sets, repetitions, and weight $(\mathrm{kg})$ ] has previously been used as a means of calculating resistance training loads (Peterson et al. 2011). The volume load for each participant was standardised across each protocol, with all sets, repetitions, and external weight being used in the calculation. The volume load value was then divided by training duration in minutes (previously used in the calculation of session perceived load) to calculate training efficiency $\left(\mathrm{kg} \mathrm{min}{ }^{-1}\right)$. This method of calculating resistance training efficiency has previously been used (Robbins et al. 2010a, c).

\section{Statistical analyses}

Data are presented as mean \pm standard deviation (SD) or standardised effect size (ES) $\pm 90 \%$ confidence intervals (90\% CI). Prior to analysis, all data were log transformed to reduce bias arising from non-uniformity error, and then analysed for practical significance using magnitude-based inferences (Batterham and Hopkins 2006). The threshold for a change to be considered to meet the smallest worthwhile change/difference $($ SWC/D) was set at $0.2 \times$ the between participant SD and was calculated using an online spreadsheet (Hopkins 2006b). For between group comparisons (e.g., TRAD vs. SS), the standardised ES of the variables being analysed were compared to assess the magnitude of difference between the two protocols (Hopkins 2006a). The probability that the magnitude of change or difference was greater than the SWC/D was rated as $<0.5 \%$, almost certainly not; 0.5-5\%, very unlikely; 5-25\%, unlikely; 25-75\%, possibly; 75-95\%, likely; 95-99.5\%, very likely; $>99.5 \%$, almost certainly (Hopkins et al. 2009). Where the $90 \%$ confidence interval (CI) crossed both the upper and lower boundaries of the SWC/D $(\mathrm{ES} \pm 0.2)$, the magnitude of change was described as unclear (Hopkins et al. 2009). ES thresholds were set at $<0.2$ (trivial), 0.2-0.6 (small), 
0.6-1.2 (large), and 1.2-2.0 (very large) (Hopkins et al. 2009).

\section{Results}

\section{Perceived exertion, volume load, and efficiency}

The mean \pm SD for time, RPE, session perceived load, and efficiency $\left(\mathrm{kg} \mathrm{min}^{-1}\right)$ of each protocol are presented in Table 2. In addition, inferences and ES $( \pm 90 \% \mathrm{CI})$ of between condition comparisons are supplied.

\section{Blood lactate concentration}

Figure 2 presents mean \pm SD [Lac] and between group comparisons across the three protocols. [Lac] preexercise was: TRAD, $1.30 \pm 0.50 ; \mathrm{SS}, 1.50 \pm 0.46$; TRI, $1.40 \pm 0.34 \mathrm{mmol} \mathrm{l}^{-1}$. At the completion of set 6 [Lac] was: TRAD, $7.90 \pm 1.50$; SS, $9.40 \pm 1.60$; TRI, $10.40 \pm 1.98 \mathrm{mmol}^{-1}$. Between group comparisons showed likely to almost certain differences between protocols $(\mathrm{ES} \pm 90 \% \mathrm{CI}$; TRAD vs. $\mathrm{SS}=0.96 \pm 0.51$, SS vs. TRI $=0.59 \pm 0.56$, TRAD vs. TRI $=1.50 \pm 0.51)$. At the completion of set, 12 [Lac] was: TRAD, $8.50 \pm 2.30$; SS, $10.70 \pm 2.14$; TRI, $14.10 \pm 2.96 \mathrm{mmol} \mathrm{l}^{-1}$. Between group comparisons showed very likely to almost certain differences between protocols $(\mathrm{ES} \pm 90 \% \mathrm{CI}$; TRAD vs. $\mathrm{SS}=0.92 \pm 0.60, \mathrm{SS}$ vs. $\mathrm{TRI}=1.42 \pm 0.57$, TRAD vs. TRI $=2.03 \pm 0.51$ ). Immediately, post-exercise [Lac] was: TRAD, $7.40 \pm 1.48$; SS, $11.50 \pm 2.19$; TRI, $13.40 \pm 1.74 \mathrm{mmol} \mathrm{l}^{-1}$. Between group comparisons showed very likely to almost certain differences between

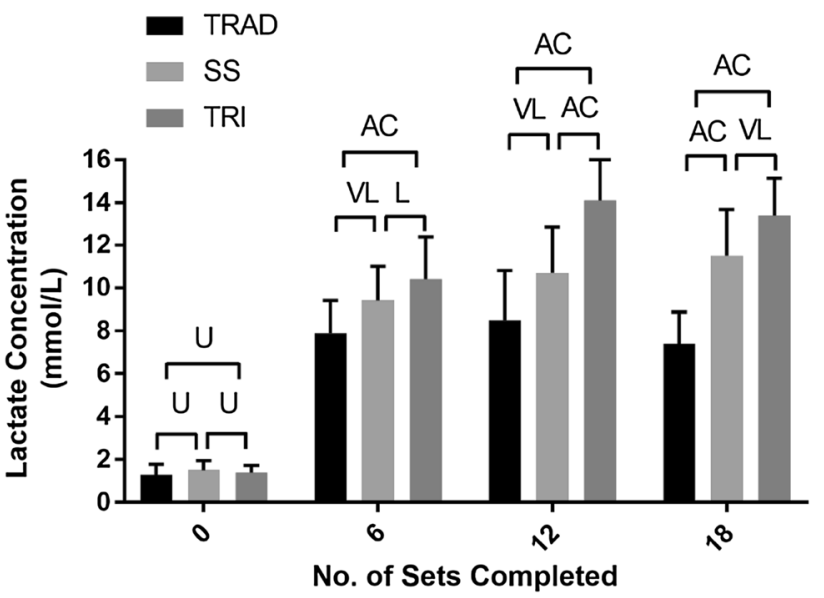

Fig. 2 Blood lactate responses throughout a traditional, superset, or tri-set resistance training protocol. $U$ unclear, $L$ likely, $V L$ very likely, $A C$ almost certain

protocols $(\mathrm{ES} \pm 90 \% \mathrm{CI}$; TRAD vs. $\mathrm{SS}=2.33 \pm 0.74, \mathrm{SS}$ vs. $\mathrm{TRI}=0.84 \pm 0.48, \mathrm{TRAD}$ vs. $\mathrm{TRI}=3.14 \pm 0.54$ ).

\section{Countermovement jump}

Table 3 presents the mean \pm SD performance of each CMJ variable at pre-, post-, and 24-h post-exercise. Furthermore, percentage change $( \pm 90 \% \mathrm{CI})$, standardised ES $( \pm 90 \% \mathrm{CI})$, and inference of change between timepoints are provided. Figure 3 presents the standardised ES $( \pm 90 \%$ CI) change from pre- to post-exercise, and pre- to 24-h post-exercise. In addition, between condition comparisons of ES change are presented as ES $( \pm 90 \% \mathrm{CI})$ and inference.

Table 2 Mean \pm SD reported time, RPE, session perceived load, and efficiency, and between condition comparison of traditional (TRAD), superset (SS), and tri-set (TRI) resistance training protocols

\begin{tabular}{|c|c|c|c|c|c|c|}
\hline & $\begin{array}{l}\text { TRAD } \\
\text { Mean } \pm \text { SD }\end{array}$ & $\begin{array}{l}\text { SS } \\
\text { Mean } \pm \text { SD }\end{array}$ & $\begin{array}{l}\text { TRI } \\
\text { Mean } \pm \text { SD }\end{array}$ & $\begin{array}{l}\text { TRAD vs. SS } \\
\text { Inference } \\
\mathrm{ES} \pm 90 \% \mathrm{CI}\end{array}$ & $\begin{array}{l}\text { SS vs. TRI } \\
\text { Inference } \\
\mathrm{ES} \pm 90 \% \mathrm{CI}\end{array}$ & $\begin{array}{l}\text { TRAD vs. TRI } \\
\text { Inference } \\
\mathrm{ES} \pm 90 \% \mathrm{CI}\end{array}$ \\
\hline Time (min) & $42.3 \pm 1.3$ & $24.0 \pm 1.2$ & $17.7 \pm 1.6$ & $\begin{array}{l}\text { TRAD }>\text { SS } \\
\text { Almost certainly } \\
15.68 \pm 0.67\end{array}$ & $\begin{array}{l}\mathrm{SS}>\mathrm{TRI} \\
\text { Almost certainly } \\
8.46 \pm 0.84\end{array}$ & $\begin{array}{l}\text { TRAD }>\text { TRI } \\
\text { Almost certainly } \\
24.14 \pm 1.11\end{array}$ \\
\hline RPE (AU) & $4.4 \pm 2.2$ & $6.8 \pm 1.2$ & $7.6 \pm 0.8$ & $\begin{array}{l}\text { TRAD }<\text { SS } \\
\text { Almost certainly } \\
1.05 \pm 0.36\end{array}$ & $\begin{array}{l}\text { SS }<\text { TRI } \\
\text { Possibly } \\
0.27 \pm 0.21\end{array}$ & $\begin{array}{l}\text { TRAD }<\text { TRI } \\
\text { Almost certainly } \\
1.32 \pm 0.43\end{array}$ \\
\hline Session perceived load (AU) & $184.2 \pm 92.1$ & $163.1 \pm 34.3$ & $134.9 \pm 17.7$ & $\begin{array}{l}\text { TRAD > SS } \\
\text { Possibly } \\
0.28 \pm 0.34\end{array}$ & $\begin{array}{l}\mathrm{SS}>\mathrm{TRI} \\
\text { Very likely } \\
0.72 \pm 0.39\end{array}$ & $\begin{array}{l}\text { TRAD > TRI } \\
\text { Very likely } \\
0.44 \pm 0.17\end{array}$ \\
\hline Efficiency $\left(\mathrm{kg} \mathrm{min}^{-1}\right)$ & $275.0 \pm 52.3$ & $483.3 \pm 106.3$ & $656.5 \pm 144.4$ & $\begin{array}{l}\text { TRAD }<\text { SS } \\
\text { Almost certainly } \\
3.10 \pm 0.13\end{array}$ & $\begin{array}{l}\mathrm{SS}<\mathrm{TRI} \\
\text { Almost certainly } \\
1.67 \pm 0.16\end{array}$ & $\begin{array}{l}\text { TRAD }<\text { TRI } \\
\text { Almost certainly } \\
4.78 \pm 0.22\end{array}$ \\
\hline
\end{tabular}

$T R A D$ traditional protocol, $S S$ superset protocol, TRI tri-set protocol, Time average time in minutes to complete each protocol, $R P E$ rate of perceived exertion of each protocol, Efficiency the mean number of kilograms lifted per minute, Min minutes, Mean $\pm S D$ mean \pm standard deviation, $E S \pm 90 \% C I$ standardised effect size $\pm 90 \%$ confidence interval 
Table 3 CMJ variable responses from pre-, post-, and 24-h post- a traditional (TRAD), superset (SS), and tri-set (TRI) resistance training protocol

\begin{tabular}{|c|c|c|c|c|c|c|c|c|c|}
\hline & $\begin{array}{l}\text { Pre- } \\
M \pm S D\end{array}$ & $\begin{array}{l}\text { Post- } \\
\mathrm{M} \pm \mathrm{SD}\end{array}$ & $\begin{array}{l}\text { Pre-Post } \\
\% \Delta \pm 90 \% \\
\text { CI }\end{array}$ & $\begin{array}{l}\text { Pre-Post } \\
\mathrm{ES} \pm 90 \% \mathrm{CI}\end{array}$ & $\begin{array}{l}\text { Pre-Post } \\
\text { Inference }\end{array}$ & $\begin{array}{l}\text { Post } 24 \text { h- } \\
M \pm S D\end{array}$ & $\begin{array}{l}\text { Pre-24h } \\
\% \Delta \pm 90 \% \\
\text { CI }\end{array}$ & $\begin{array}{l}\text { Pre-24h } \\
\mathrm{ES} \pm 90 \% \mathrm{CI}\end{array}$ & $\begin{array}{l}\text { Pre-24 h } \\
\text { Inference }\end{array}$ \\
\hline \multicolumn{10}{|l|}{ TRAD } \\
\hline $\begin{array}{l}\text { Jump } \\
\text { height } \\
(\mathrm{cm})\end{array}$ & $37.9 \pm 6.1$ & $35.5 \pm 6.0$ & $-6.2 \pm 2.5$ & $-0.41 \pm 0.17$ & Very Likely $\downarrow$ & $37.9 \pm 6.8$ & $0.10 \pm 3.0$ & $0.01 \pm 0.19$ & Likely $\leftrightarrow$ \\
\hline FT:CT & $0.65 \pm 0.15$ & $0.65 \pm 0.17$ & $-0.20 \pm 4.9$ & $-0.01 \pm 0.22$ & Unclear & $0.68 \pm 0.14$ & $4.1 \pm 4.4$ & $0.18 \pm 0.19$ & Possible $\uparrow$ \\
\hline $\begin{array}{l}\left.\mathrm{PP} / \mathrm{BM}^{-1}\right) \\
\quad\left(\mathrm{W} \mathrm{kg}^{-1}\right)\end{array}$ & $55.3 \pm 8.3$ & $53.1 \pm 7.4$ & $-3.9 \pm 1.5$ & $-0.27 \pm 0.10$ & Likely $\downarrow$ & $55.3 \pm 8.3$ & $0.0 \pm 2.0$ & $0.00 \pm 0.13$ & $\begin{array}{l}\text { Very Likely } \\
\leftrightarrow\end{array}$ \\
\hline \multicolumn{10}{|l|}{ SS } \\
\hline $\begin{array}{l}\text { Jump } \\
\text { height } \\
(\mathrm{cm})\end{array}$ & $38.9 \pm 6.2$ & $37.5 \pm 5.6$ & $-3.7 \pm 3.1$ & $-0.24 \pm 0.20$ & Possible $\downarrow$ & $37.2 \pm 6.0$ & $-4.5 \pm 2.9$ & $-0.29 \pm 0.19$ & Likely $\downarrow$ \\
\hline FT:CT & $0.64 \pm 0.15$ & $0.65 \pm 0.14$ & $1.0 \pm 3.1$ & $0.04 \pm 0.13$ & $\begin{array}{l}\text { Very Likely } \\
\stackrel{\leftrightarrow}{ }\end{array}$ & $0.63 \pm 0.18$ & $-1.3 \pm 3.3$ & $-0.06 \pm 0.15$ & Likely $\leftrightarrow$ \\
\hline $\begin{array}{l}\left.\mathrm{PP} / \mathrm{BM}^{-1}\right) \\
\quad\left(\mathrm{W} \mathrm{kg}^{-1}\right)\end{array}$ & $55.1 \pm 6.6$ & $54.7 \pm 7.7$ & $-0.90 \pm 2.8$ & $-0.07 \pm 0.23$ & Likely $\leftrightarrow$ & $53.7 \pm 7.5$ & $-2.7 \pm 1.8$ & $-0.22 \pm 0.15$ & Likely $\downarrow$ \\
\hline \multicolumn{10}{|l|}{ TRI } \\
\hline $\begin{array}{l}\text { Jump } \\
\text { height } \\
(\mathrm{cm})\end{array}$ & $38.3 \pm 6.1$ & $37.1 \pm 6.7$ & $-3.10 \pm 2.4$ & $-0.20 \pm 0.16$ & Possible $\downarrow$ & $37.7 \pm 6.4$ & $-1.6 \pm 2.7$ & $-0.10 \pm 0.18$ & Likely $\leftrightarrow$ \\
\hline FT:CT & $0.67 \pm 0.14$ & $0.67 \pm 0.12$ & $0.20 \pm 6.3$ & $0.01 \pm 0.31$ & Unclear & $0.65 \pm 0.14$ & $-3.9 \pm 4.9$ & $-0.20 \pm 0.25$ & Possible $\downarrow$ \\
\hline $\begin{array}{l}\left.\mathrm{PP} / \mathrm{BM}^{-1}\right) \\
\left(\mathrm{W} \mathrm{kg}^{-1}\right)\end{array}$ & $55.5 \pm 10.0$ & $54.5 \pm 8.2$ & $-1.8 \pm 3.4$ & $-0.10 \pm 0.19$ & Likely $\leftrightarrow$ & $53.6 \pm 8.6$ & $-3.3 \pm 3.4$ & $-0.19 \pm 0.17$ & Possible $\downarrow$ \\
\hline
\end{tabular}

$M \pm S D$ mean \pm standard deviation, $\% \Delta$ percentage change, $E S$ effect size, $90 \% C I 90 \%$ confidence interval, $F T: C T$ flight time:contraction time, $P P / B M$ peak power per kilogram of body mass, $\uparrow$ increase, $\leftrightarrow$ trivial, $\downarrow$ decrease

\section{Testosterone and cortisol concentration}

Table 4 presents mean \pm SD mean value of testosterone and cortisol concentration at pre-, post-, and 24-h post-exercise. Furthermore, percentage change $( \pm 90 \% \mathrm{CI})$, standardised ES $( \pm 90 \% \mathrm{CI})$, and inference of change between timepoints are provided. Figure 4 presents the standardised ES $( \pm 90 \%$ CI) change from pre- to post-exercise, and pre- to $24-\mathrm{h}$ post-exercise. In addition, between condition comparison of ES change is presented as ES ( $\pm 90 \% \mathrm{CI})$ and inference.

\section{Creatine kinase concentration}

Table 5 presents mean \pm SD, percentage change $( \pm 90 \%$ CI), standardised ES $( \pm 90 \% \mathrm{CI})$, and inference of change between timepoints of [CK] at pre-, post-, and 24-h postexercise. Furthermore, between condition comparisons of ES change are presented as ES $( \pm 90 \% \mathrm{CI})$ and inference.

\section{Discussion}

This is the first study to compare the acute and short-term physiological responses (i.e., metabolic, neuromuscular, and endocrine) between TRAD, SS, and TRI resistance training set structures. SS and TRI protocols were almost certainly more efficient (i.e., kilograms lifted per minute) than TRAD, with possible to likely lower session perceived load. Immediately post-exercise, TRAD training showed the greatest decrease in CMJ performance. However, at 24-h post-training, the TRAD protocol showed trivial or possibly improved neuromuscular function, while SS and TRI protocols still showed possible or likely reductions. During and immediately after the resistance training protocols, SS and TRI had very likely to almost certainly greater [Lac], with the TRI protocol being likely to almost certainly greater than the SS. At $24 \mathrm{~h}$, changes in $[\mathrm{CK}]$ were likely trivial in the TRAD protocol, but very likely and likely increased after the SS and TRI, respectively. Testosterone was possibly greater immediately post-exercise in the SS and TRI, while at 24-h post-training, only SS remained possibly greater. Cortisol responses were almost certainly and likely reduced in the TRAD and SS conditions, respectively, but at $24 \mathrm{~h}$, SS showed possible increases, while TRAD remained likely reduced. These outcomes indicate that SS and TRI resistance training can enhance training efficiency, but also cause differing perceived and physiological responses. 


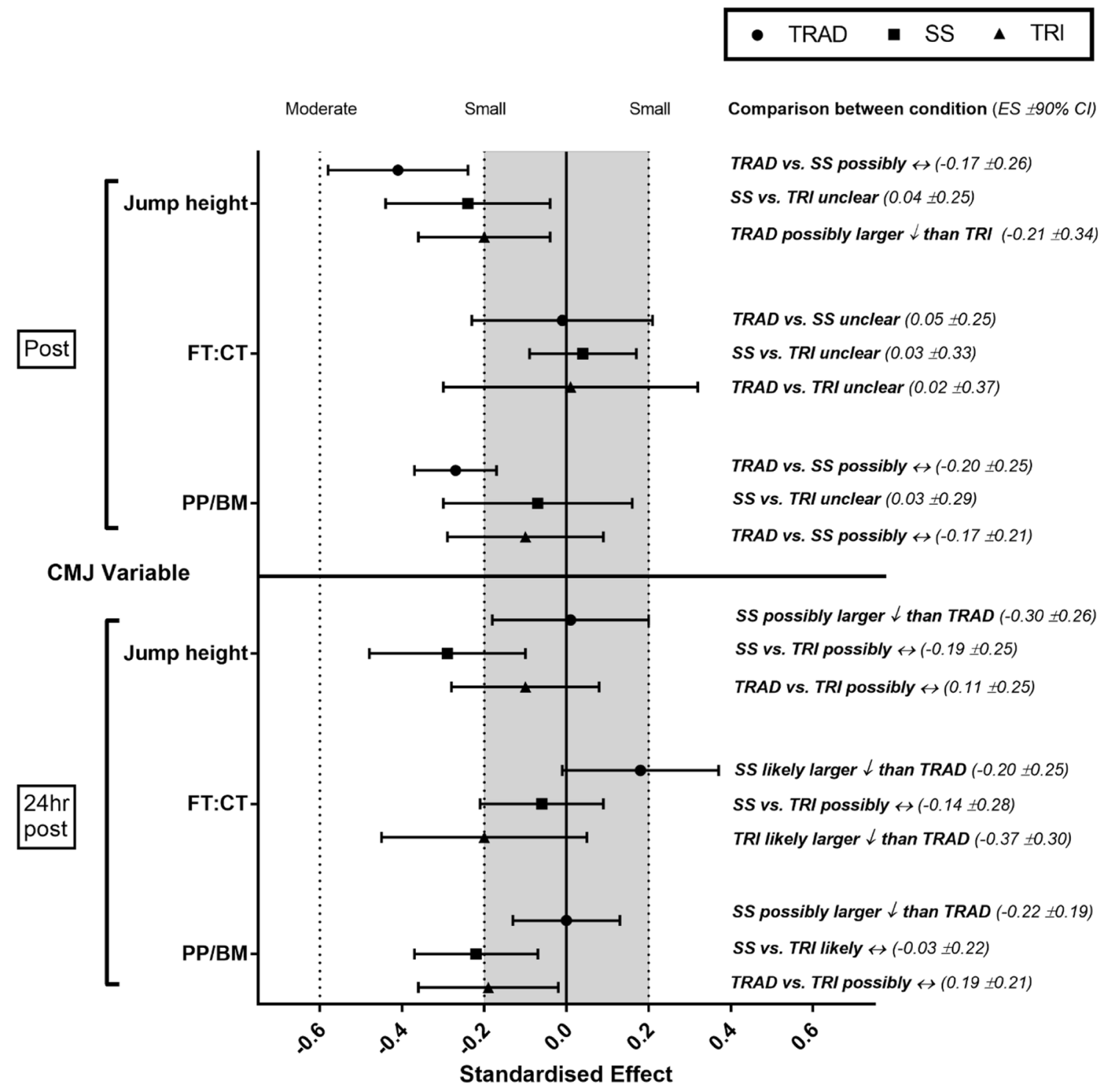

Fig. 3 Standardised effect size $( \pm 90 \%$ CI $)$ changes and inferences of between condition comparisons of CMJ variables immediately and $24 \mathrm{~h}$ after traditional (TRAD), superset (SS), and tri-set (TRI) resistance training

\section{Perceived exertion, training load, and efficiency}

Findings showed that as training efficiency increased, there are associated increases in RPE and decreases in session perceived load. For session intensity, findings are consistent with previous research showing that increased training efficiency coincides with increases in perceived exertion (Hiscock et al. 2017). However, when factored with time (i.e., session perceived load), SS and TRI may induce lower perceived total training loads (refer to Table 2). While previous research has not directly investigated the effects of resistance training efficiency on session perceived load, it should be noted that protocols that increase training efficiency have indicated lower total session perceived load
(Hiscock et al. 2017). This suggests that session perceived load may not be sensitive to increases in resistance training efficiency when intensity and volume are held constant and highlight the relative shortcomings of this method of athlete monitoring. Therefore, session perceived load may not be optimal when used as a sole measure of resistance training monitoring. Accordingly, it is proposed that the practitioner and sport scientist account for training load efficiency (i.e., $\mathrm{kg} \mathrm{min}^{-1}$ ) in conjunction with RPE and/ or session perceived load when implementing these resistance training methods. This may assist in the monitoring of resistance training and the physiological responses (e.g., neuromuscular function and metabolic changes) that can occur in response to enhanced resistance training efficiency. 
Table 4 Testosterone ( $\mathrm{pg} \mathrm{ml}^{-1}$ ) and cortisol ( $\mathrm{ng} \mathrm{ml}^{-1}$ ) responses from pre-, post-, and 24-h post- a traditional (TRAD), superset (SS), and tri-set (TRI) resistance training protocol

\begin{tabular}{|c|c|c|c|c|c|c|c|c|c|}
\hline & $\begin{array}{l}\text { Pre- } \\
M \pm S D\end{array}$ & $\begin{array}{l}\text { Post- } \\
\mathrm{M} \pm \mathrm{SD}\end{array}$ & $\begin{array}{l}\text { Pre-Post } \\
\% \Delta \pm 90 \% \text { CI }\end{array}$ & $\begin{array}{l}\text { Pre-Post } \\
\mathrm{ES} \pm 90 \% \mathrm{CI}\end{array}$ & $\begin{array}{l}\text { Pre-Post } \\
\text { Inference }\end{array}$ & $\begin{array}{l}\text { Post } 24 \mathrm{~h}- \\
\mathrm{M} \pm \mathrm{SD}\end{array}$ & $\begin{array}{l}\text { Pre }-24 \mathrm{~h} \\
\% \Delta \pm 90 \% \\
\text { CI }\end{array}$ & $\begin{array}{l}\text { Pre-24h } \\
\mathrm{ES} \pm 90 \% \mathrm{CI}\end{array}$ & $\begin{array}{l}\text { Pre-24 h } \\
\text { Inference }\end{array}$ \\
\hline \multicolumn{10}{|c|}{ Testosterone $\left(\mathrm{pg} \mathrm{ml}^{-1}\right)$} \\
\hline TRAD & $139.6 \pm 76.8$ & $138.5 \pm 55.4$ & $-0.70 \pm 10.7$ & $-0.02 \pm 0.23$ & Unclear & $135.9 \pm 84.3$ & $-2.6 \pm 18.4$ & $-0.06 \pm 0.40$ & Unclear \\
\hline SS & $132.8 \pm 67.8$ & $143.7 \pm 74.7$ & $8.2 \pm 4.9$ & $0.18 \pm 0.29$ & Possible $\uparrow$ & $150.3 \pm 61.6$ & $-13.1 \pm 20.3$ & $0.28 \pm 0.41$ & Possible $\uparrow$ \\
\hline TRI & $127.0 \pm 68.6$ & $133.9 \pm 71.0$ & $-5.4 \pm 12.4$ & $0.11 \pm 0.26$ & Possible $\uparrow$ & $124.4 \pm 61.0$ & $-2.1 \pm 15.3$ & $-0.05 \pm 0.34$ & Unclear \\
\hline \multicolumn{10}{|c|}{ Cortisol (ng ml ${ }^{-1}$ ) } \\
\hline TRAD & $0.21 \pm 0.23$ & $0.10 \pm 0.07$ & $-50.29 \pm 10.90$ & $-0.88 \pm 0.27$ & $\begin{array}{c}\text { Almost cer- } \\
\quad \text { tain } \downarrow\end{array}$ & $0.16 \pm 0.18$ & $-22.0 \pm 16.7$ & $-0.31 \pm 0.27$ & Likely $\downarrow$ \\
\hline SS & $0.20 \pm 0.21$ & $0.14 \pm 0.17$ & $-28.33 \pm 22.40$ & $-0.43 \pm 0.40$ & Likely $\downarrow$ & $0.25 \pm 0.22$ & $27.1 \pm 40.4$ & $0.31 \pm 0.41$ & Possible $\uparrow$ \\
\hline TRI & $0.17 \pm 0.24$ & $0.15 \pm 0.13$ & $-14.10 \pm 33.00$ & $-0.16 \pm 0.40$ & Unclear & $0.18 \pm 0.20$ & $5.6 \pm 30.2$ & $0.06 \pm 0.30$ & Unclear \\
\hline
\end{tabular}

$M \pm S D$ mean \pm standard deviation, $\% \Delta$ percentage change, ES effect size, $90 \% C I 90 \%$ confidence interval, $\uparrow$ increase, $\downarrow$ decrease

Fig. 4 Standardised effect size $( \pm 90 \% \mathrm{CI})$ change of testosterone and cortisol immediately post- and $24 \mathrm{~h}$ after traditional (TRAD), superset (SS), and tri-set (TRI) resistance training. Also presented is the standardised effect size $( \pm 90 \% \mathrm{CI})$ and inference of comparisons between condition of testosterone and cortisol immediately and $24 \mathrm{~h}$ after exercise. $\downarrow$, decrease; $\uparrow$, increase
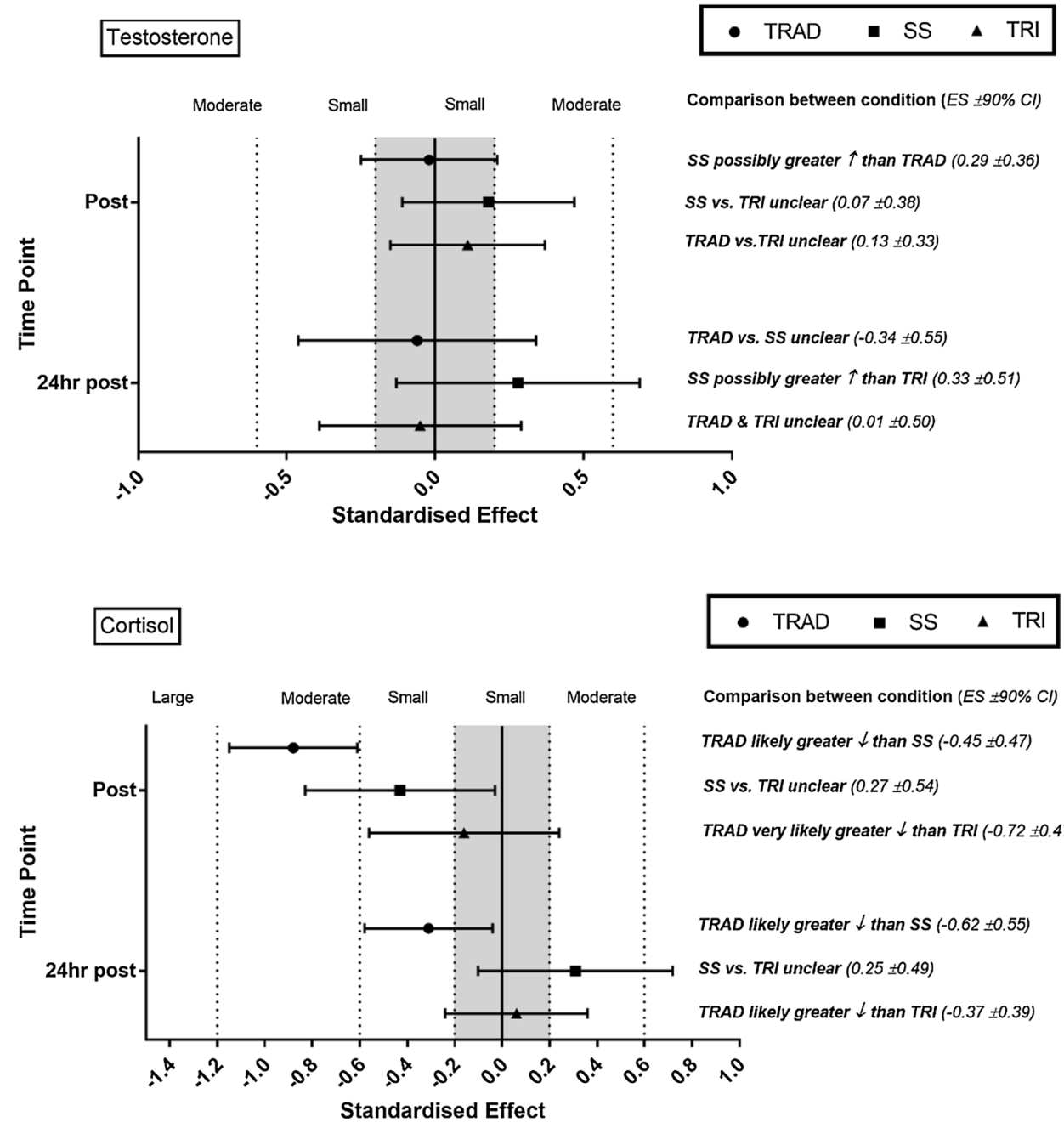

\section{Neuromuscular responses}

While results in the current study indicated that the TRAD protocol had the lowest mean RPE, likely and very likely reductions in $\mathrm{PP} / \mathrm{BM}$ and jump height occurred immediately post-exercise. Increased RPE has previously been related to larger immediate reductions in CMJ function (Hiscock et al. 2017). However, the current study shows 
Table 5 Creatine kinase concentration $\left(\mathrm{U}^{-1}\right)$ change and between condition comparison pre- and post-24 h after a traditional (TRAD), superset (SS), and tri-set (TRI) resistance training protocol

\begin{tabular}{|c|c|c|c|c|c|c|}
\hline & $\begin{array}{l}\text { Pre- } \\
\mathrm{M} \pm \mathrm{SD}\left(\mathrm{U}^{-1}\right)\end{array}$ & $\begin{array}{l}\text { Post } 24 \mathrm{~h}- \\
\mathrm{M} \pm \mathrm{SD}\left(\mathrm{U}^{-1}\right)\end{array}$ & $\begin{array}{l}\text { Pre-24h } \\
\% \Delta \pm 90 \% \mathrm{CI}\end{array}$ & $\begin{array}{l}\text { Pre- } 24 \mathrm{~h} \\
\mathrm{ES} \pm 90 \% \mathrm{CI}\end{array}$ & $\begin{array}{l}\text { Pre-24 h } \\
\text { Inference }\end{array}$ & $\begin{array}{l}\text { Between condition comparison } \\
\mathrm{ES} \pm 90 \% \mathrm{CI} \text { and inference }\end{array}$ \\
\hline TRAD & $264.9 \pm 227.8$ & $286.6 \pm 292.3$ & $8.2 \pm 13.7$ & $0.12 \pm 0.19$ & Likely $\leftrightarrow$ & $\begin{array}{l}0.43 \pm 0.29 \\
\text { SS likely greater increases than TRAD }\end{array}$ \\
\hline SS & $248.9 \pm 179.2$ & $355.2 \pm 202.4$ & $42.7 \pm 20.4$ & $0.62 \pm 0.23$ & Very Likely $\uparrow$ & $\begin{array}{l}0.16 \pm 0.38 \\
\text { Differences between SS and TRI unclear }\end{array}$ \\
\hline TRI & $262.3 \pm 183.6$ & $326.1 \pm 247.8$ & $24.40 \pm 22.2$ & $0.39 \pm 0.32$ & Likely $\uparrow$ & $\begin{array}{l}0.27 \pm 0.36 \\
\text { TRI possibly greater increases than TRAD }\end{array}$ \\
\hline
\end{tabular}

$M \pm S D$ mean \pm standard deviation, \% $\Delta$ percentage change, $E S$ effect size, $90 \%$ CI 90\% confidence interval, $\uparrow$ increase, $\leftrightarrow$ trivial

that the TRAD protocol had the largest decrease in performance post-training. The $-6.2 \% \pm 2.5$ decrease in jump height was unexpected, particularly when compared with the smaller changes in the SS $(-3.7 \% \pm 3.1)$ and TRI $(-3.1 \% \pm 2.4)$ protocols, and may occur due to the additional rest time provided in the TRAD protocol. This additional time was thought to maximise recovery and minimise residual fatigue. Nevertheless, at $24 \mathrm{~h}$, neuromuscular function of the lower body in the TRAD protocol appeared to have recovered, while the SS and TRI conditions still showed possible or likely reductions in performance. It would, therefore, be plausible that the immediate decrease in CMJ observed in the TRAD protocol was attributed to surplus rest causing "cooling down" of the participant's lower body musculature (i.e., participants completing the TRAD protocol had not exercised the lower body for approximately $25 \mathrm{~min}$ due to altered exercise order and recovery periods compared to approximately $12 \mathrm{~min}$ in the TRI protocol) and/or a lack of potentiation due to the relatively light loading (Sale 2004). This reflects the findings of Hiscock et al. (2017) who found that resistance training protocols that include additional rest and are of a lower RPE may demonstrate improved recovery of neuromuscular function at 24-h post-exercise. This suggests that additional recovery time within a resistance training session may diminish the effects of fatigue on neuromuscular performance.

Results in this study also indicated that enhanced training efficiency can affect the duration of reduced neuromuscular function. At 24-h post-training, the SS protocol had likely decreases in both jump height and PP/BM, whereas TRI had possible decreases in FT:CT and PP/ BM. Recent research suggests that FT:CT may indicate altered CMJ strategy during a fatigued state (Rowell et al. 2016), while relative peak power output has demonstrated very large relationships $(r=0.81)$ with ballistic capabilities (Hori et al. 2008). By assessing not only CMJ height but also CMJ variables, it is possible that an improved understanding of fatigue responses can occur (Gathercole et al. 2015). It is conceivable, therefore, that the suppressed neuromuscular function reported at $24 \mathrm{~h}$ is indicative of fatigue responses that would not be detected when only assessing CMJ height. However, this measure of neuromuscular function could not distinguish between training protocols that have very high volume loads per minute (i.e., SS and TRI). Nevertheless, the practitioner should be aware of the effects of enhanced training efficiency on performance. It would also be prudent for the practitioner and scientist to incorporate relative (e.g., PP/BM ) and time-derived (e.g., FT:CT) measures of neuromuscular function (Gathercole et al. 2015). These measures may provide additional understanding of fatigue responses that basic output measures (e.g., jump height) may not be able to detect (Gathercole et al. 2015).

\section{Metabolic responses}

Alternative structuring of resistance training which eliminates rest periods between exercises is known to increase the anaerobic requirements which impact upon metabolic perturbation and fatigue (Kelleher et al. 2010). This is demonstrated in the current study by the protocols that have the highest efficiency (i.e., SS and TRI) demonstrating the greatest rises in peak lactate (Fig. 2). With increased lactate production, an accumulation of hydrogen ions occurs. This accumulation is thought to interfere with muscle excitation and contraction coupling via calcium binding to troponin (Devries et al. 1982; Sahlin et al. 1997; Vasquez et al. 2013), causing a reduction in functional capacity of muscle fibres and an increased emphasis on the motor cortex to signal the recruitment of additional larger motor units (Houtman et al. 2003; Sahlin et al. 1997; Vollestad et al. 1984). It is thought that these changes can also lead to elevations in RPE and reduced power output (Hardee et al. 2012). Therefore, by reducing recovery time within resistance training sessions, the practitioner can expect decreased neuromuscular performance, and increased metabolic responses (e.g., [LAC]) and perceived measures of intensity. Furthermore, activation and recruitment of the musculature may change 
due to increased motor unit signalling required to maintain neuromuscular performance (McCaulley et al. 2009).

As well as increased lactate accumulation, our results show that enhanced efficiency can influence indices of muscle damage (Table 5). While no studies to date have investigated SS or TRI training structures on changes in [CK], research by Mayhew and colleagues (2005) assessed the effects of rest interval length (i.e., 1 vs. $3 \mathrm{~min}$ ) in volume equated resistance training protocols. Their findings corroborate the current study, showing reduced session recovery can impact upon [CK] responses. This provides further evidence that additional recovery within resistance training protocols can attenuate muscle damage. It has been suggested that these augmented [CK] responses are in part due to reductions in recovery causing increased metabolic stress within the muscle (Hiscock et al. 2017). Corresponding increases in ammonia and hydrogen ions can lead to reduced cellular integrity and damage to contractile elements of the muscle fibres (Sanchez-Medina and GonzálezBadillo 2011; Váczi et al. 2013). This damage has also been linked to impaired force-generating ability which may have impacted upon 24-h CMJ performance (Hiscock et al. 2017; Váczi et al. 2013).

\section{Endocrine responses}

Despite previously documented relationships between [LAC] accumulation and testosterone secretion (Walker et al. 2011), the current study did not show this. Changes in testosterone were either unclear or showed a possible small response across all timepoints and protocols. These responses may have been due to the underlying makeup of the SS and TRI structures. A number of criteria are essential for a substantial acute rise in testosterone due to resistance exercise (e.g., young, well-trained, male athletes, and completing high volumes of resistance training in workouts that have shortened recovery periods) (Hooper et al. 2017), with the current study fulfilling a number of these criteria. However, previous work by Linnamo et al. (2005) showed that certain intensity thresholds (i.e., $\geq 75 \%$ of $1 \mathrm{RM}$ ) are also required to elicit acute testosterone responses, with the current study being below this threshold (i.e., $60 \%$ of $1 \mathrm{RM})$. While increases in resistance training intensity may prove beneficial in eliciting testosterone responses, this may not have been possible in practice due to the large amounts of fatigue and metabolic perturbation that participants recorded when completing the SS and TRI protocols. Nevertheless, this inability of SS and TRI resistance training to induce substantial changes in acute testosterone responses may impede upregulation of the androgen receptor and corresponding downstream genomic responses (Hooper et al. 2017). It should also be noted, however, that due to the large variance in individual responses, findings must be interpreted with caution. In stating this, it can be said that TRAD resistance training with $65 \%$ of $3 \mathrm{RM}$ load promotes an almost certain reduction in salivary cortisol. This indicates that TRAD resistance training, that incorporates increased recovery time, may exert a smaller neuroendocrine stress response when exercising at the same intensity when compared with SS and TRI (Crewther et al. 2017).

While this study is the first to examine the physiological responses to SS and TRI training structures, it is not without limitations. First, resistance training intensity and volume between all three protocols (i.e., TRAD, SS, and TRI) were matched. While this is a methodological strength that assists in the examination and comparison of the three protocols, it may limit transferability to real life practice. Due to the differing amount of recovery time provided within each session, recovery was facilitated to differing extents. Increased recovery is known to prolong time to concentric failure in subsequent resistance training sets (Schoenfeld et al. 2016) and improve the maintenance of training intensity (Willardson and Burkett 2008). Therefore, the TRAD and SS regimes may have been able to tolerate greater intensities or volumes. Consequently, research comparing TRAD, SS, and TRI protocols in circumstances that manipulate these resistance training variables is warranted. Second, the current findings only extended over a 24-h time frame. Increased training efficiency has previously been linked to suppressed neuromuscular function at 48-h post-exercise (Hiscock et al. 2017), and it is still unknown at what timepoint neuromuscular function returned to baseline after completing the SS and TRI training protocols. While physiological changes over this short time frame may imply potential responses and adaptations, further research is needed to quantify the effects of these resistance training programmes.

\section{Conclusion}

In conclusion, SS and TRI methodologies are efficient means of resistance training compared to TRAD alone. However, with these, improvements in efficiency come notable changes in perceptions of training intensity, muscle damage, and within-session lactate concentrations. These increases in metabolic measures occur simultaneously with likely to very likely reductions in neuromuscular performance at 24-h post-training which indicates a greater fatigue response. Endocrine responses in the current study varied widely. However, TRAD structures induced a smaller neuroendocrine stress response immediately and $24 \mathrm{~h}$ after training.

The practitioner should consider utilising SS and TRI resistance training methods during time-constrained 
periods or when other training outcomes require increased training time (e.g., skill development). Alternatively, these methods could be used as a form of metabolic conditioning when an improvement in total training capacity is desired due to the large metabolic responses (e.g., [Lac]) that occur. However, with this enhanced training, efficiency comes a possible need for increased recovery. Consequently, the practitioner may wish to place SS and TRI forms of training at the beginning of the training week to assist in the management of fatigue. This could be further developed by placing TRAD protocols towards the latter half of the training week due to the smaller suppression of neuromuscular function at 24-h post-training.

\section{Compliance with ethical standards}

Conflict of interest The authors declare that they have no conflict of interest.

Open Access This article is distributed under the terms of the Creative Commons Attribution 4.0 International License (http:// creativecommons.org/licenses/by/4.0/), which permits unrestricted use, distribution, and reproduction in any medium, provided you give appropriate credit to the original author(s) and the source, provide a link to the Creative Commons license, and indicate if changes were made.

\section{References}

Baldari C, Bonavolonta V, Emerenziani GP, Gallotta MC, Silva AJ, Guidetti L (2009) Accuracy, reliability, linearity of Accutrend and Lactate Pro versus EBIO Plus analyzer. Eur J Appl Physiol 107:105-111. doi:10.1007/s00421-009-1107-5

Balsamo S et al (2012) Exercise order affects the total training volume and the ratings of perceived exertion in response to a super-set resistance training session. Int J Gen Med 5:123-127. doi:10.2147/IJGM.S27377

Batterham AM, Hopkins WG (2006) Making meaningful inferences about magnitudes. Int J Sports Physiol Perform 1:50-57. doi:10.1123/ijspp.1.1.50

Crewther BT, Cook C (2010) Measuring the salivary testosterone and cortisol concentrations of weightlifters using an enzymeimmunoassay kit. Int J Sports Med 31:486-489. doi:10.105 5/s-0030-1249619

Crewther BT, Thomas AG, Stewart-Williams S, Kilduff LP, Cook CJ (2017) Is salivary cortisol moderating the relationship between salivary testosterone and hand-grip strength in healthy men? Eur J Sport Sci 17:188-194. doi:10.1080/17461391.2016.1220628

Darrall-Jones JD, Jones B, Till K (2015) Anthropometric and physical profiles of English academy rugby union players. J Strength Cond Res 29:2086-2096. doi:10.1519/jsc.0000000000000872

Devries HA, Moritani T, Nagata A, Magnussen K (1982) The relation between critical power and neuromuscular fatigue as estimated from electromyographic data. Ergonomics 25:783-791. doi: $10.1080 / 00140138208925034$

Foster C et al (2001) A new approach to monitoring exercise training. J Strength Cond Res 15:109-115. doi:10.1519/00124278-200102000-00019

Gathercole R, Sporer B, Stellingwerff T, Sleivert G (2015) Alternative countermovement-jump analysis to quantify acute neuromuscular fatigue. Int J Sports Physiol Perform 10:84-92. doi:10.1123/ijspp.2013-0413

Gaviglio CM, Crewther BT, Kilduff LP, Stokes KA, Cook CJ (2014) Relationship between pregame concentrations of free testosterone and outcome in rugby union. Int J Sports Physiol Perform 9:324-331. doi:10.1123/ijspp.2013-0106

Haddad HA, Simpson BM, Buchheit M (2015) Monitoring changes in jump and sprint performance: best or average values? Int J Sports Physiol Perform 10:931-934. doi:10.1123/ijspp.2014-0540

Haff GG, Triplett NT (2015) Essentials of strength training and conditioning, 4th edn. Human Kinetics, Leeds

Hardee JP, Lawrence MM, Utter AC, Triplett NT, Zwetsloot KA, McBride JM (2012) Effect of inter-repetition rest on ratings of perceived exertion during multiple sets of the power clean. Eur J Appl Physiol 112:3141-3147. doi:10.1007/s00421-011-2300-x

Harries SK, Lubans DR, Callister R (2016) Comparison of resistance training progression models on maximal strength in sub-elite adolescent rugby union players. J Sci Med Sport 19:163-169. doi:10.1016/j.jsams.2015.01.007

Hiscock DJ, Dawson B, Clarke M, Peeling P (2017) Can changes in resistance exercise workload influence internal load, countermovement jump performance and the endocrine response? J Sports Sci. doi:10.1080/02640414.2017.1290270

Hooper DR, Kraemer WJ, Focht BC, Volek JS, DuPont WH, Caldwell LK, Maresh CM (2017) Endocrinological roles for testosterone in resistance exercise responses and adaptations. Sports Med. doi:10.1007/s40279-017-0698-y

Hopkins WG (2006a) A spreadsheet for combining outcomes from several subject groups. Sportscience 10:50-53. http://www. sportsci.org/2006/wghcom.htm. Accessed 26 Nov 2016

Hopkins WG (2006b) Spreadsheets for analysis of controlled trials with adjustment for a predictor Sportscience 10:46-50. http:// www.sportsci.org/2006/wghcontrial.htm. Accessed 26 Nov 2016

Hopkins W, Marshall S, Batterham A, Hanin J (2009) Progressive statistics for studies in sports medicine and exercise science. Med Sci Sports Exerc 41:3-13. doi:10.1249/MSS.0b013e31818cb278

Hori N, Newton RU, Andrews WA, Kawamori N, McGuigan MR, Nosaka K (2008) Does performance of hang power clean differentiate performance of jumping, sprinting, and changing of direction? J Strength Cond Res 22:412-418. doi:10.1519/ JSC.0b013e318166052b

Houtman CJ, Stegeman DF, Van Dijk JP, Zwarts MJ (2003) Changes in muscle fiber conduction velocity indicate recruitment of distinct motor unit populations. J Appl Physiol 95:1045-1054. doi:10.1152/japplphysiol.00665.2002

Kelleher AR, Hackney KJ, Fairchild TJ, Keslacy S, Ploutz-Snyder LL (2010) The metabolic costs of reciprocal supersets vs. traditional resistance exercise in young recreationally active adults. J Strength Cond Res 24:1043-1051. doi:10.1519/ JSC.0b013e3181d3e993

Linnamo V, Pakarinen A, Komi PV, Kraemer WJ, Hakkinen K (2005) Acute hormonal responses to submaximal and maximal heavy resistance and explosive exercises in men and women. J Strength Cond Res 19:566-571. doi:10.1519/r-15404.1

Maia MF, Willardson JM, Paz GA, Miranda H (2014) Effects of different rest intervals between antagonist paired sets on repetition performance and muscle activation. J Strength Cond Res 28:2529-2535. doi:10.1519/jsc.0000000000000451

Mayhew DL, Thyfault JP, Koch AJ (2005) Rest-interval length affects leukocyte levels during heavy resistance exercise. J Strength Cond Res 19:16-22. doi:10.1519/r-14113.1

McCaulley GO, McBride JM, Cormie P, Hudson MB, Nuzzo JL, Quindry JC, Travis Triplett N (2009) Acute hormonal and neuromuscular responses to hypertrophy, strength and power type resistance exercise. Eur J Appl Physiol 105:695-704. doi:10.1007/s00421-008-0951-z 
McGuigan M (2017) Monitoring training and performance in athletes. Human Kinetics, Leeds

Pareja-Blanco F et al (2016) Effects of velocity loss during resistance training on athletic performance, strength gains and muscle adaptations. Scand J Med Sci Sports. doi:10.1111/sms.12678

Peterson MD, Pistilli E, Haff GG, Hoffman EP, Gordon PM (2011) Progression of volume load and muscular adaptation during resistance exercise. Eur J Appl Physiol 111:1063-1071. doi:10.1007/s00421-010-1735-9

Phibbs PJ et al (2017) Organised chaos in late specialisation team sports: weekly training loads of elite adolescent rugby union players. J Strength Cond Res. doi:10.1519/ jsc.0000000000001965

Robbins DW, Young WB, Behm DG (2010a) The effect of an upperbody agonist-antagonist resistance training protocol on volume load and efficiency. J Strength Cond Res 24:2632-2640. doi:10.1519/JSC.0b013e3181e3826e

Robbins DW, Young WB, Behm DG, Payne WR (2010b) Agonistantagonist paired set resistance training: a brief review. J Strength Cond Res 24:2873-2882. doi:10.1519/JSC.0b013e3181f00bfc

Robbins DW, Young WB, Behm DG, Payne WR (2010c) The effect of a complex agonist and antagonist resistance training protocol on volume load, power output, electromyographic responses, and efficiency. J Strength Cond Res 24:1782-1789. doi:10.1519/ JSC.0b013e3181dc3a53

Roe G, Darrall-Jones J, Till K, Phibbs P, Read D, Weakley J, Jones B (2016a) Between-day reliability and sensitivity of common fatigue measures in rugby players. Int J Sports Physiol Perform 11:581-586. doi:10.1123/ijspp.2015-0413

Roe G, Till K, Darrall-Jones J, Phibbs P, Weakley J, Read D, Jones B (2016b) Changes in markers of fatigue following a competitive match in elite academy rugby union players. S Afr $\mathbf{J}$ sports med 28:1-4. doi:10.17159/2078-516X/2016/v28i1a418

Rowell AE, Aughey RJ, Hopkins WG, Stewart AM, Cormack SJ (2016) Identification of sensitive measures of recovery following external load from football match play. Int J Sports Physiol Perform. doi:10.1123/ijspp.2016-0522

Sabido R, Peñaranda M, Hernández-Davó JL (2016) Comparison of acute responses to four different hypertrophy-oriented resistance training methodologies. Hum Mov Sci 37:109-121

Sahlin K, Soderlund K, Tonkonogi M, Hirakoba K (1997) Phosphocreatine content in single fibers of human muscle after sustained submaximal exercise. Am J Physiol 273:C172-C178

Sale D (2004) Postactivation potentiation: role in performance. Br J Sports Med 38:386-387. doi:10.1136/bjsm.2002.003392

Sanchez-Medina L, González-Badillo JJ (2011) Velocity loss as an indicator of neuromuscular fatigue during resistance training. Med Sci Sports Exerc 43:1725-1734. doi:10.1249/ MSS.0b013e318213f880

Schoenfeld BJ (2010) The mechanisms of muscle hypertrophy and their application to resistance training. J Strength Cond Res 24:2857-2872. doi:10.1519/JSC.0b013e3181e840f3

Schoenfeld B (2011) The use of specialized training techniques to maximize muscle hypertrophy. Strength Cond J 33:60-65. doi:10.1519/SSC.0b013e3182221ec2
Schoenfeld BJ et al (2016) Longer interset rest periods enhance muscle strength and hypertrophy in resistance-trained men. J Strength Cond Res 30:1805-1812. doi:10.1519/JSC.0000000000001272

Singh F, Foster C, Tod D, McGuigan MR (2007) Monitoring different types of resistance training using session rating of perceived exertion. Int J Sports Physiol Perform 2:34-45. doi:10.1123/ ijspp.2.1.34

Smart DJ, Gill ND (2013) Effects of an off-season conditioning program on the physical characteristics of adolescent rugby union players. J Strength Cond Res 27:708-717. doi:10.1519/ JSC.0b013e31825d99b0

Till K, Jones B, Darrall-Jones J, Emmonds S, Cooke C (2015) Longitudinal development of anthropometric and physical characteristics within academy rugby league players. J Strength Cond Res 29:1713-1722. doi:10.1519/JSC.0000000000000792

Till K, Darrall-Jones J, Weakley J, Roe G, Jones B (2016) The influence of training age on the annual development of physical qualities within academy rugby league players. J Strength Cond Res. doi: $10.1519 /$ jsc.0000000000001546

Uribe BP, Coburn JW, Brown LE, Judelson DA, Khamoui AV, Nguyen D (2010) Muscle activation when performing the chest press and shoulder press on a stable bench vs. a Swiss ball. J Strength Cond Res 24:1028-1033. doi:10.1519/ JSC.0b013e3181ca4fb8

Váczi M, Rácz L, Hortobágyi T, Tihanyi J (2013) Dynamic contractility and efficiency impairments in stretch-shortening cycle are stretch-load-dependent after training-induced muscle damage. J Strength Cond Res 27:2171-2179. doi:10.1519/ JSC.0b013e31827da32c

Vasquez LM, McBride JM, Paul JA, Alley JR, Carson LT, Goodman CL (2013) Effect of resistance exercise performed to volitional failure on ratings of perceived exertion. Percept Mot Skills 117:881-891. doi:10.2466/27.29.PMS.117x30z8

Vollestad NK, Vaage O, Hermansen L (1984) Muscle glycogen depletion patterns in type I and subgroups of type II fibres during prolonged severe exercise in man. Acta Physiol Scand 122:433-441. doi:10.1111/j.1748-1716.1984.tb07531.x

Walker S, Taipale RS, Nyman K, Kraemer WJ, Häkkinen K (2011) Neuromuscular and hormonal responses to constant and variable resistance loadings. Med Sci Sports Exerc 43:26-33. doi:10.1249/MSS.0b013e3181e71bcb

Weakley J, Till K, Darrall-Jones J, Roe G, Phibbs P, Read D, Jones B (2017a) The influence of resistance training experience on the between-day reliability of commonly used strength measures in male youth athletes. J Strength Cond Res. doi:10.1519/ jsc. 0000000000001883

Weakley JJS, Till K, Darrall-Jones J, Roe GAB, Phibbs PJ, Read DB, Jones BL (2017b) Strength and conditioning practices in adolescent rugby players: relationship with changes in physical qualities. J Strength Cond Res. doi:10.1519/jsc.0000000000001828

Willardson JM, Burkett LN (2008) The effect of different rest intervals between sets on volume components and strength gains. J Strength Cond Res 22:146-152. doi:10.1519/ JSC.0b013e31815f912d 